\title{
Evaluation of pulmonary disease using static lung volumes in primary ciliary dyskinesia
}

\author{
Massimo Pifferi, ${ }^{1}$ Andrew Bush, ${ }^{2}$ Giovanni Pioggia, ${ }^{3}$ Davide Caramella, ${ }^{4}$ \\ Gennaro Tartarisco, ${ }^{3}$ Maria Di Cicco, ${ }^{1}$ Marta Zangani ${ }^{4}$ Iolanda Chinellato, ${ }^{5}$ \\ Fabrizio Maggi, ${ }^{6}$ Giovanna Tezza, ${ }^{5}$ Pierantonio Macchia, ${ }^{1}$ Attilio Boner ${ }^{5}$
}

\begin{abstract}
- Additional materials are published online only. To view these files please visit the journal online (http://dx.doi.org/ 10.1136/thoraxjnl-2011200137).

${ }^{1}$ Department of Paediatrics, University of Pisa, Pisa, Italy ${ }^{2}$ Imperial School of Medicine at the National Heart and Lung Institute, London, UK

${ }^{3}$ Institute of Clinical Physiology, CNR, Pisa, Italy

${ }^{4}$ Department of Diagnostic and Interventional Radiology, University of Pisa, Pisa, Italy ${ }^{5}$ Department of Paediatrics, University of Verona, Verona, Italy

${ }^{6}$ Department of Experimental Pathology, University of Pisa, Pisa, Italy
\end{abstract}

\section{Correspondence to}

Dr Massimo Pifferi, University of Pisa, Department of Pediatrics, Via Roma 67, Pisa 56126, Italy; m.pifferi@med.unipi.it

Received 5 March 2011 Revised 1 June 2012 Accepted 15 June 2012

Published Online First 7 July 2012

\section{ABSTRACT}

Background In primary ciliary dyskinesia (PCD) lung damage is usually evaluated by high-resolution CT (HRCT).

Objective To evaluate whether HRCT abnormalities and Pseudomonas aeruginosa infection were better predicted by spirometry or plethysmography.

Methods A cross-sectional study performed in consecutive patients with PCD who underwent sputum culture, spirometry, plethysmography and HRCT within $48 \mathrm{~h}$. Principal component analysis and soft computing were used for data evaluation.

Results Fifty patients (26 children) were studied. $P$ aeruginosa infection was found in $40 \%$ of the patients and bronchiectasis in $88 \%$. There was a correlation between infection with $P$ aeruginosa and extent of bronchiectasis ( $p=0.009 ; r=0.367)$ and air-trapping $(p=0.03 ; r=0.315)$. Moreover, there was an association between infection with $P$ aeruginosa and residual volume (RV) values $>150 \%(p=0.04)$ and RV/total lung capacity (TLC) ratio $>140 \%$ ( $p=0.001)$, but not between infection with $P$ aeruginosa and forced expiratory volume in $1 \mathrm{~s}\left(\mathrm{FEV}_{1}\right)<80 \%$, or forced expiratory flow between $25 \%$ and $75 \%$ of forced vital capacity (FVC) $\left(\mathrm{FEF}_{25-75 \%}\right)<70 \%$ or $\mathrm{FEV}_{1} / \mathrm{FVC}<70 \%(<80 \%$ in children). Severity of the total lung impairment on chest HRCT directly correlated with RV when expressed as per cent predicted $(p=0.003 ; r=0.423)$, and RV $/ \mathrm{TLC}$ $(p<0.001 ; r=0.513)$ or when expressed as $z$ scores $(p=0.002, r=0.451$ and $p<0.001, r=0.536$ respectively). Principal component analysis on plethysmographic but not on spirometry data allowed recognition of different severities of focal air trapping, atelectasis and extent of bronchiectasis.

Conclusions Plethysmography better predicts HRCT abnormalities than spirometry. Whether it might be a useful test to define populations of patients with PCD who should or should not have HRCT scans requires further longitudinal studies.

\section{INTRODUCTION}

In primary ciliary dyskinesia (PCD), abnormal motility of respiratory cilia and impaired mucociliary clearance result in recurrent infections of the airways, eventually causing permanent parenchymal damage, and progressive decline in lung function. ${ }^{1}$

Chest high-resolution CT (HRCT) has become the method of choice to evaluate structural airway
Key messages

What is the key question?

- Do lung volume measurements predict lung damage better than spirometry in patients with primary ciliary dyskinesia?

What is the bottom line?

- Raised residual volume and residual volume/ total lung capacity ratio are associated with Pseudomonas aeruginosa infection and with lung damage shown by high-resolution CT.

\section{Why read on?}

- The evaluation of static volumes by plethysmography seems to be an underappreciated method for the follow-up of children with primary ciliary dyskinesia; early identification of deterioration in such parameters could result in a more adequate treatment of the disease and in research of eventual unhealthy attitudes of the patients.

changes, ${ }^{2}$ and is used in a number of chronic respiratory diseases including PCD. ${ }^{34}$

However, there is no evidence that regular CT scans affect outcome in $\mathrm{PCD}^{5}$ and the potential lifetime cumulative radiation exposure should be considered. ${ }^{67}$

PCD guidelines suggest that lung function should be evaluated at every visit in cooperative children ${ }^{1} 5{ }^{8}$ since it has been shown that the severity of structural abnormalities may correlate with impairment in forced expiratory volume in $1 \mathrm{~s}$ $\left(\mathrm{FEV}_{1}\right){ }^{3}{ }^{4}$ However, a decreased $\mathrm{FEV}_{1}$ is not indicative of the site of airway obstruction ${ }^{9}$ and, as in cystic fibrosis, ${ }^{10}$ there are preliminary suggestions that PCD is characterised by marked peripheral airway dysfunction. ${ }^{11}$ Since small airways obstruction may lead to air trapping and to a consequent increase in residual volume (RV) and RV/ total lung capacity (TLC) ratio, ${ }^{9}$ we hypothesised that raised functional residual capacity (FRC) made by plethysmography (FRCpleth), and RV and/or RV/TLC ratio, indirect calculated estimates of pulmonary hyperinflation and lung restriction respectively, may be more sensitive than spirometry to structural changes as shown by HRCT in PCD. The aim of the study was to determine the 
correlations between HRCT abnormalities and dynamic or static lung function parameters in patients with PCD to assess their potential use as clinical monitoring tools in PCD. ${ }^{12}$

\section{MATERIALS AND METHODS Subjects}

Between March 2008 and May 2010, we enrolled all consecutively newly diagnosed patients with PCD aged $\geq 6$ years followed up in the Department of Paediatrics of the University of Pisa. PCD was diagnosed on standard criteria. ${ }^{1}$ We obtained sputum, cough swab or pharyngeal aspirate cultures, spirometry, body plethysmography and a HRCT of the chest. A sputum culture was collected, preferably by expectoration. Pharyngeal aspirate was obtained in uncooperative children after an overnight fast using a disposable catheter connected to a mucus extractor inserted into the mouth to a depth of $7-10 \mathrm{~cm}$ and drawn back while applying gentle suction.

Informed consent was obtained from adult patients or from the parents of children. The study protocol was approved by the local Hospital Ethical Committee.

\section{Ciliary motion analysis and ultrastructural studies}

Samples were obtained from the inferior turbinate using a cytology brush (Microvasive, Milford, Massachusetts, USA) for immediate light microscopy, transmission electron microscopy and cell cultures. ${ }^{13}$ Ciliary morphology, motion pattern, beat frequency, ultrastructural studies and ciliary activity after ciliogenesis in culture were evaluated. ${ }^{14-16}$

Ciliary motion analysis, ultrastructural assessment and cultures were performed by different operators each one blind to the results obtained by the others.

\section{Lung function}

All lung function testing (Master Screen Body equipment; Jaeger, Wuerzburg, Germany) was performed by experienced doctors (MP, MDC) utilising standard American Thoracic Society methodology. ${ }^{17}{ }^{18} \mathrm{FEV}_{1}$, forced vital capacity (FVC), forced expiratory flow between $25 \%$ and $75 \%$ of FVC $\left(\mathrm{FEF}_{25-75 \%}\right)$, FRCpleth, RV, TLC, RV/TLC and parameters of airway mechanics including airway resistance (Raw), specific airway resistance (sRaw) and effective specific resistance (sReff) were expressed as percentage of predicted and $z$ scores values. ${ }^{19}$ For plethysmography in each patient we obtained at least three reproducible manoeuvres. To be accepted, single inspiratory manoeuvres needed to have yielded virtually superimposable X-Y plots, and values of FRCpleth had to be within $5 \%$ of each other. ${ }^{20}$

Lung function studies were all performed on the same day, within 2 days of the HRCT.

\section{CT scanning of chest}

In all patients chest HRCT was performed using the same scanner (Multislice CT; General Electric Medical Systems, Milwaukee, Michigan, USA). Slices (1 mm thick) were obtained with $10 \mathrm{~mm}$ spacing $(100-120 \mathrm{kV}, 80-130 \mathrm{~mA})$, in the supine position. All images were evaluated by the same radiologist (DC) who was blinded to the clinical data and scored using a modified Bhalla system, ${ }^{21}$ which includes severity of bronchiectasis (score $0-3)$ and extent of bronchiectasis (score $0-3$ ), mucous plugging (score $0-3$ ), peribronchial thickening (score $0-3$ ), parenchymal abnormalities, such as atelectasis (score 0-3) and focal airtrapping (score $0-3$ ). Bronchiectasis was identified according to standard criteria. ${ }^{22}$
Thus, a severity class (from 1 to 3 ) for total lung impairment was obtained (class of severity 1 for total score of $0-6$, class 2 for total score of 7-12, class 3 for total score of 13-18).

\section{Soft computing analysis of data}

Data were analysed with soft computing methodologies. Basic elements of soft computing and the application of intelligent control have been recently introduced. ${ }^{23}$ The soft computingbased modelling approach was applied to either flow-volume data or body plethysmography or both to develop a model predictive of chest HRCT scores. The model was identified by means of a self-organising artificial neural network (ANN). ANNs are mathematical models in which distributed adaptable parameters are modified through a learning process according to real data. Kohonen self-organising map (KSOM) predictive models were identified to classify chest HRCT scores, (total lung impairment, severity of bronchiectasis, extent of bronchiectasis, peribronchial thickening, mucous plugging, atelectasis, focal airtrapping), starting from flow-volume parameters (FEV1, FVC $\mathrm{FEV}_{1}$ /FVC, $\mathrm{FEF}_{25-75 \%}$ ), body plethysmography data (FRCpleth, RV, TLC, RV/TLC) and parameters of airway mechanics (Raw, sRaw, sReff). To check the generalisation capability of the neural network, a 10-fold cross-validation process was carried out. In this work, we fixed a $5 \times 5$ neurons KSOM with the parameters $\alpha(T)=0.8$ and a training of 5000 epochs, which allowed us to obtain the best performance of the model. Further details on KSOM ANNs are reported in the supplementary material online.

\section{Statistical analyses}

The principal component analysis (PCA) methodology was applied to display data. PCA is a mathematical linear transformation aimed at reducing the dimensionality of (number of variables in) a dataset to a manageable level while retaining as much as possible of the variance present in the original dataset. ${ }^{24}$ Data from correlated groups of variables are used to calculate a smaller number of uncorrelated variables (principal components) to simplify further analyses. We used the eigenvalue decomposition ${ }^{24}$ to obtain the first three principal components in our dataset (denoted Y1, Y2 and Y3) which are associated with $99 \%$ of the variance.

There are no data in the published literature to inform a power calculation, so the sample size was opportunistic. Baseline variables were expressed as group mean \pm SD or as median and IOR when the variables were non-normally distributed. Moreover, to allow comparison of observations from different normal distributions, all lung function data were expressed as SD (z) scores. ${ }^{25} Z$-score transformation was carried out by using the equation $z=(x-\mu) / \sigma$, where $x$ is the raw score or observation to be standardised, $\mu$ is the mean of the reference population, and $\sigma$ is the $\mathrm{SD}$ of the reference population. Differences between means and distributions were evaluated by the two-tailed Student t test.

Correlations between continuous non-normally distributed variables were assessed using Spearman's rank correlation coefficients. One-way analysis of variance (ANOVA) comparing the values of plethysmography indexes between the severity class for total lung impairment at HRCT was also applied. The $\chi^{2}$ test was used to evaluate the association between Pseudomonas aeruginosa (alone or with other bacterial infection) and RV $>150 \%$, RV/TLC ratio $>140 \%$ and $\mathrm{FEV}_{1}<80 \%$ or FEF $25-75 \%$ $<70 \%$ of predicted. The association between $P$ aeruginos $a$ and $\mathrm{FEV}_{1} / \mathrm{FVC}<80 \%$ predicted for children and $<70 \%$ predicted for adults was also evaluated. These cut-offs were selected because 
Table 1 Spirometry and plethysmography parameters expressed as per cent of predicted and $z$ scores

\begin{tabular}{|c|c|c|c|c|}
\hline \multirow{2}{*}{$\begin{array}{l}\text { Functional } \\
\text { parameters }\end{array}$} & \multicolumn{2}{|c|}{$\%$ of predicted } & \multicolumn{2}{|l|}{ z Scores } \\
\hline & Range & Mean (SD) & Range & Mean (SD) \\
\hline $\mathrm{FEV}_{1}$ & $31.0-136.0$ & $85.3(24.6)$ & $-2.82-1.47$ & $-0.60(1.0)$ \\
\hline FVC & $47.0-138.0$ & $93.4(18.5)$ & $-2.86-2.05$ & $-0.35(1.0)$ \\
\hline $\mathrm{FEF}_{25 / 75}$ & $10.0-151.0$ & $63.2(34.4)$ & $-2.63-1.49$ & $-1.07(1.0)$ \\
\hline $\mathrm{FEV}_{1} / \mathrm{FVC}$ & $42.0-98.8$ & 75.45 (13.35) & $-2.86-1.08$ & $-0.67(1.0)$ \\
\hline Raw & $55.4-310.6$ & $164.5(62.9)$ & $-0.71-3.35$ & $1.02(1.0)$ \\
\hline sRaw & $71.0-739.2$ & $269.0(142.0)$ & $-0.20-4.50$ & $1.19(1.0)$ \\
\hline sReff & $64.0-680.7$ & $240.6(128.1)$ & $-0.28-4.53$ & $1.09(1.0)$ \\
\hline FRC & $101.2-248.0$ & $159.3(31.2)$ & $-0.04-4.74$ & $1.90(1.0)$ \\
\hline RV & $83.0-397.0$ & $196.5(69.4)$ & $-0.24-4.28$ & $1.39(1.0)$ \\
\hline TLC & $85.0-174.0$ & $119.2(16.7)$ & $-0.89-4.46$ & $1.15(1.0)$ \\
\hline RV/TLC & $91.0-305.0$ & $161.0(45.6)$ & $-0.20-4.49$ & $1.34(1.0)$ \\
\hline
\end{tabular}

$\mathrm{FEV}_{1}$, forced expiratory volume in $1 \mathrm{~s}$; FRC, functional residual capacity; FVC, forced vital capacity; Raw, airway resistance; RV, residual volume; sRaw, specific airway resistance; sReff, effective specific resistance; TLC, total lung capacity.

they were previously demonstrated to correlate with air-trapping on HRCT in children. ${ }^{26}$

A $p$ value $<0.05$ was considered statistically significant. All statistical calculations were performed using SPSS V.18.0 for Windows (XP/Vista/7).

\section{RESULTS}

Fifty patients were studied. Twenty-six were children (19 boys and seven girls, age range 6-17 years, median 11 years; IOR 5.25 ) and 24 adults (nine men and 15 women, age range 18-47 years, median 30.5 years; IOR 9.5).

Infection with $P$ aeruginosa (with or without other bacteria) was found in $20(40.0 \%)$ patients, seven of whom were children (27\% infected). Other organisms (Serratia marcescens, Rhodococcus equi, Streptococcus pneumoniae, Staphylococcus aureus, Enterobacter cloacae, Streptococcus pyogenes, Alcaligenes xylosoxidans) were found in $12(24.0 \%)$ subjects.

Results of spirometry and plethysmography expressed as per cent of predicted and as $z$ scores are reported in table 1.

HRCT Bhalla score was 0 in 3 subjects, 1 in 12, 2 in 27, and 3 in the remaining 8 subjects. Bronchiectasis was documented in $44(88 \%)$ patients, 21 of whom were children. Bronchiectasis was present in multiple lobes in $36 / 44$ (82\%) subjects. The distribution of bronchiectasis is detailed in table 2 .

Patient age was inversely correlated with per cent predicted $\mathrm{FEV}_{1} \quad(\mathrm{p}=0.006 ; \mathrm{r}=-0.384)$, FVC $(\mathrm{p}=0.04 ; \mathrm{r}=-0.295)$, $\mathrm{FEF}_{25-75 \%}(\mathrm{p}=0.01 ; \mathrm{r}=-0.362)$ and $\mathrm{FEV}_{1} / \mathrm{FVC}(\mathrm{p}=0.02 ;$ $\mathrm{r}=-0.336$ ) but surprisingly not with FRCpleth, RV, TLC and $\mathrm{RV} / \mathrm{TLC}$. There was no correlation between patient age and Raw, sRaw or sReff per cent predicted.
Table 2 Bronchiectasis distribution (\%) in multiple lobes

\begin{tabular}{lll}
\hline Lobes & Right & Left \\
\hline Upper & 20 & 25 \\
Middle & 45 & $11.4^{*}$ \\
Lower & 84 & 70 \\
Lingula & $6.8^{*}$ & 16 \\
\hline
\end{tabular}

*In patients with situs inversus.

Considering z scores, patient age was inversely correlated with FEV $_{1} \quad(p=0.038 ; r=-0.294)$, with $F_{1} / F V C \quad(p=0.008$; $r=-0.372)$ and directly correlated with FVC $(p=0.001 ; r=0.451)$, with FRCpleth $(p<0.001 ; r=0.599)$, RV $(p<0.001 ; r=0.623)$, TLC $(p<0.001 ; r=0.646)$ and RV/TLC $(p=0.034 ; r=0.313)$. There was no correlation between patient age and Raw, sRaw or sReff $z$ scores.

There was a significant correlation between infection with $P$ aeruginosa and extent of bronchiectasis ( $p=0.009 ; \mathrm{r}=0.367)$ and air-trapping ( $\mathrm{p}=0.03 ; \mathrm{r}=0.315)$. Moreover, there was a significant association between infection with $P$ aeruginosa and RV values $>150 \%(p=0.04)$ and $\mathrm{RV} / \mathrm{TLC}$ ratio $>140 \%(\mathrm{p}=0.001)$, but not between infection with $P$ aeruginosa and $\mathrm{FEV}_{1}<80 \%$, or $\mathrm{FEF}_{25-75 \%}$ $<70 \%$ or $\mathrm{FEV}_{1} / \mathrm{FVC}<70 \%$ ( $<80 \%$ in children).

There were significant correlations between HRCT scores and lung function parameters. In particular, severity of the total lung impairment on chest HRCT was inversely correlated with $\mathrm{FEV}_{1}$ $(p=0.02 ; r=-0.322), F V C(p=0.007 ; r=-0.376)$, but not with FEF $25-75 \%$ or $\mathrm{FEV}_{1} / \mathrm{FVC}$, and directly correlated with RV $(p=0.003 ; \quad r=0.423) \quad$ (figure $1 \mathrm{~A})$ and RV/TLC $(p<0.001$; $\mathrm{r}=0.513$ ) (figure 2A) per cent predicted, but not with TLC. This was confirmed with lung function z scores; severity of the total lung impairment on chest HRCT directly correlated with RV $(p=0.002 ; r=0.451)$ (figure $1 B), R V / T L C(p<0.001 ; r=0.536)$ (figure $2 \mathrm{~B})$ and FRCpleth $(\mathrm{p}=0.039 ; \mathrm{r}=0.305)$, but not with TLC. The correlations between the different HRCT parameters (severity and extent of bronchiectasis, peribronchial thickening, extent of mucous plugging, consolidation or atelectasis and air trapping) and lung function results are reported in tables 3 and 4 . As can be seen there were some negative correlations between different HRCT parameters and some per cent predicted spirometry values (table 3) which disappeared when $z$ scores were analysed (table 4). By contrast the positive correlations between severity and extent of bronchiectasis and air trapping with RV and RV/TLC were dependent on whether per cent predicted (table 3) or z scores (table 4) were used.

Moreover, plethysmography indices in patients with different HRCT class severity were found to be significantly different according to one-way ANOVA ( $p=0.026$ for FRCpleth, $p=0.007$ for $R V$ and $p=0.001$ for $R V / T L C)$.
Figure 1 (A) Correlation between residual volume per cent predicted (\% pred) and total lung impairment severity score at chest high-resolution CT (HRCT). (B) Correlation between residual volume $z$ scores and total lung impairment severity score at chest HRCT.

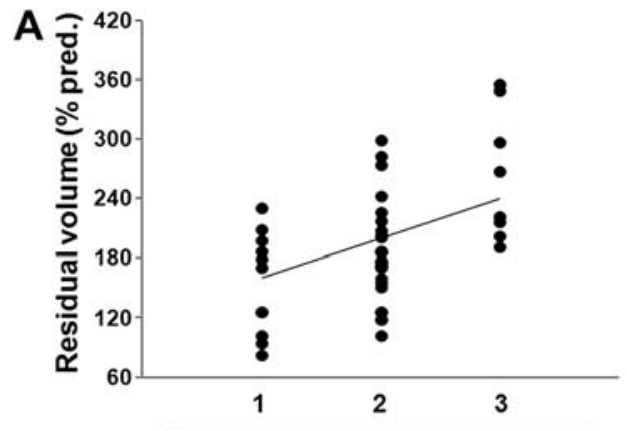

Total lung impairment at chest HRCT

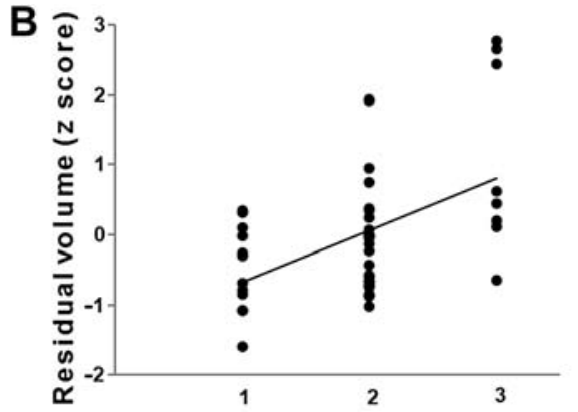

Total lung impairment at chest HRCT 
Figure 2 (A) Correlation between residual volume (RV) and total lung capacity (TLC) ratio per cent predicted (\% pred) and total lung impairment severity score at chest high-resolution CT (HRCT). (B) Correlation between RV and $T L C$ ratio $z$ scores and total lung impairment severity score at chest HRCT.

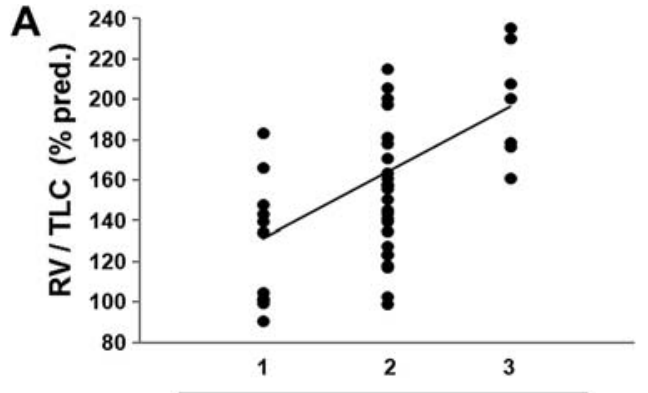

Total lung impairment at chest HRCT

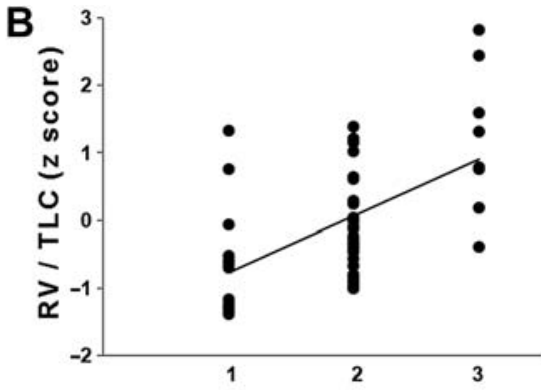

Total lung impairment at chest HRCT
Only weak or no correlations were found between the different HRCT parameters and Raw, sRaw and sReff depending on whether per cent predicted values (table 3) or z-scores (table 4) were considered.

Moreover, there was a direct correlation between the age of patients and severity of total lung impairment on chest HRCT $(p=0.002 ; r=0.425)$, and severity $(p=0.004 ; r=0.404)$ and extent of bronchiectasis $(p<0.001 ; r=0.510)$, but not with focal air-trapping scores or other parameters.

PCA performed using the body plethysmography data is depicted in figure 3 , which shows three-dimensional scatter plots of the first four principal components evaluated processing the original body plethysmography variables (FRCpleth, RV, TLC and RV/TLC ratio), after the removal of an outlier. Each scatter plot shows the differences between scores 1 and 3 for focal air-trapping (figure $3 \mathrm{~A}$ ), atelectasis (figure $3 \mathrm{~B}$ ), extent of bronchiectasis (figure $3 C$ ) and total lung impairment (figure 3D). The PCA topological study allows focal air-trapping severity, atelectasis and the extent of bronchiectasis to be visually discriminated, although the clusters overlap. The analysis does not allow severity of bronchiectasis, peribronchial thickening and mucous plugging to be discriminated. The PCA topological study of flow-volume data and of airway mechanics parameters (Raw, sRaw and sReff) does not permit any discrimination of chest HRCT scores. The PCA topological study does not identify patients with $P$ aeruginosa infection. Further results obtained by $\mathrm{KSOM}$ models are reported in the supplementary material online.

\section{DISCUSSION}

\section{Statement of principal findings}

To our knowledge this is the largest dataset of contemporaneous spirometry, plethysmography and HRCT in patients with PCD. HRCT was performed in all patients aged 6 years or older because it is known that from this age many patients already have some degree of bronchiectasis ${ }^{27}$ and from this age patients can both cooperate with the procedure and be able to perform reproducible lung function testing. ${ }^{28}$

We show that soft computing based on plethysmographic lung volumes, but not spirometry, is sensitive to a range of HRCT abnormalities. Reduction in lung function associated with bronchiectasis has been previously reported in patients with PCD. ${ }^{12} 29$ However, we report for the first time that FRCpleth, RV and RV/TLC ratio is associated with the severity of air trapping, atelectasis and extent of bronchiectasis and with $P$ aeruginosa infection. Hence follow-up of PCD patients may be preferable with plethysmography rather than simple spirometry.

\section{Strengths and weaknesses of the study}

Our study has some strengths. First the completeness of ascertainment, since all patients performed all the tests, obviating selection bias. Second all the evaluations were done within $48 \mathrm{~h}$. Third the objective analytical techniques using soft computing greatly reduces the chance of investigator bias.

\section{Strengths and weaknesses in relation to other studies}

In our study group the vast majority of adults and $80 \%$ of the children had bronchiectasis and, as previously reported, half of the adults ${ }^{29}$ and approximately one-third of the children had chronic Paeruginosa infection. The latter was associated with airtrapping, as previously documented in young children with cystic fibrosis. ${ }^{30}$ Focal air-trapping, which indicates small airways disease, probably starts early and our results document that static lung volume evaluation can detect this even in younger patients. This finding lends further support to the hypothesis that peripheral lung damage may be an early event in PCD. ${ }^{10}$

It has recently been suggested that even the youngest patients already have irreversible lung damage ${ }^{12}$ and that bronchiectasis,

Table 3 Correlation between chest high-resolution CT and functional parameters expressed as per cent of predicted normal

\begin{tabular}{|c|c|c|c|c|c|c|c|c|c|c|c|}
\hline HRCT parameters & $\mathrm{FEV}_{1}$ & FVC & $\mathrm{FEF}_{25-75 \%}$ & $\mathrm{FEV}_{1} / \mathrm{FVC}$ & Raw & sRaw & sReff & FRCpleth & RV & TLC & RV/TLC \\
\hline Severity of bronchiectasis & $\begin{array}{l}\mathrm{p}=0.01 \\
\mathrm{r}=-0.350\end{array}$ & $\begin{array}{l}\mathrm{p}=0.005 \\
\mathrm{r}=-0.393\end{array}$ & NS & NS & NS & NS & NS & NS & $\begin{array}{l}p=0.02 \\
r=0.331\end{array}$ & NS & $\begin{array}{l}p=0.005 \\
r=0.408\end{array}$ \\
\hline Extent of bronchiectasis & $\begin{array}{l}p=0.01 \\
r=-0.346\end{array}$ & $\begin{array}{l}p=0.01 \\
r=-0.356\end{array}$ & $\begin{array}{l}p=0.02 \\
r=-0.316\end{array}$ & NS & NS & NS & NS & NS & $\begin{array}{l}p=0.02 \\
r=0.336\end{array}$ & NS & $\begin{array}{l}\mathrm{p}=0.001 \\
\mathrm{r}=0.459\end{array}$ \\
\hline Peribronchial thickening & NS & NS & NS & NS & NS & NS & NS & NS & NS & NS & NS \\
\hline Extent of mucous plugging & NS & NS & NS & NS & $\begin{array}{l}\mathrm{p}=0.035 \\
\mathrm{r}=0.315\end{array}$ & $\begin{array}{l}p=0.044 \\
r=0.301\end{array}$ & NS & NS & NS & NS & NS \\
\hline
\end{tabular}

$\mathrm{FEF}_{25-75 \%}$ forced expiratory flow between $25 \%$ and $75 \%$ of forced vital capacity; FEV 1 , forced expiratory volume in 1 s; FRCpleth, functional residual capacity by plethysmography; FVC, forced vital capacity; HRCT, high-resolution CT; NS, non-significant; Raw, airway resistance; RV, residual volume; sRaw, specific airway resistance; sReff, effective specific resistance; TLC, total lung capacity. 
Table 4 Correlations between chest high-resolution CT and functional parameters expressed as z scores

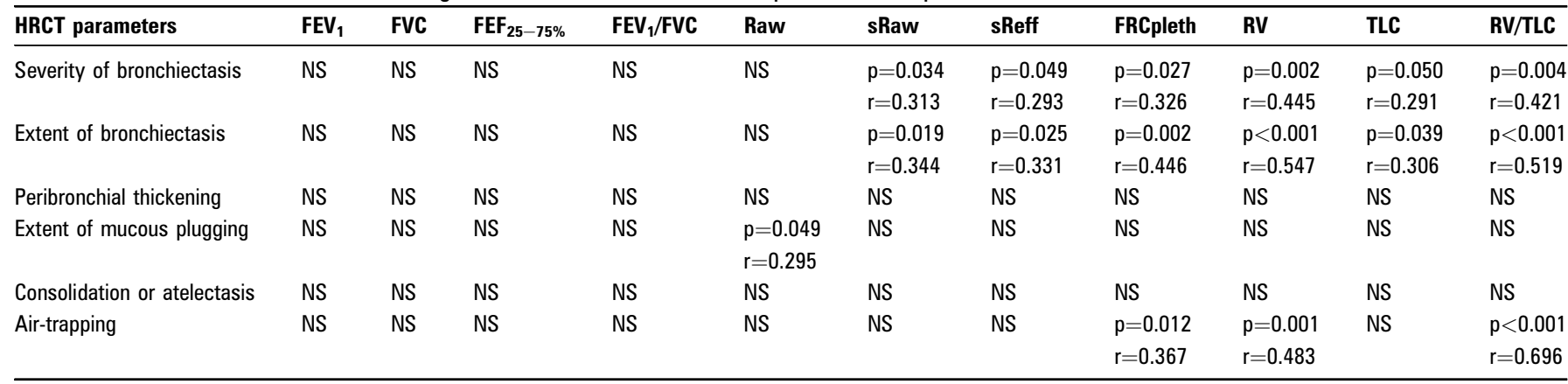

$\mathrm{FEF}_{25-75 \%}$, forced expiratory flow between $25 \%$ and $75 \%$ of forced vital capacity; FEV 1 , forced expiratory volume in $1 \mathrm{~s}$; FRCpleth, functional residual capacity by plethysmography; FVC, forced vital capacity; HRCT, high-resolution CT; NS, non-significant; Raw, airway resistance; RV, residual volume; sRaw, specific airway resistance; sReff, effective specific resistance; TLC, total lung capacity.

reduction in lung flows and increased RV and RV/TLC can be present in patients with PCD as young as $<3$ years. ${ }^{31}$ We were not able to determine this because we could not study very young children with PCD.
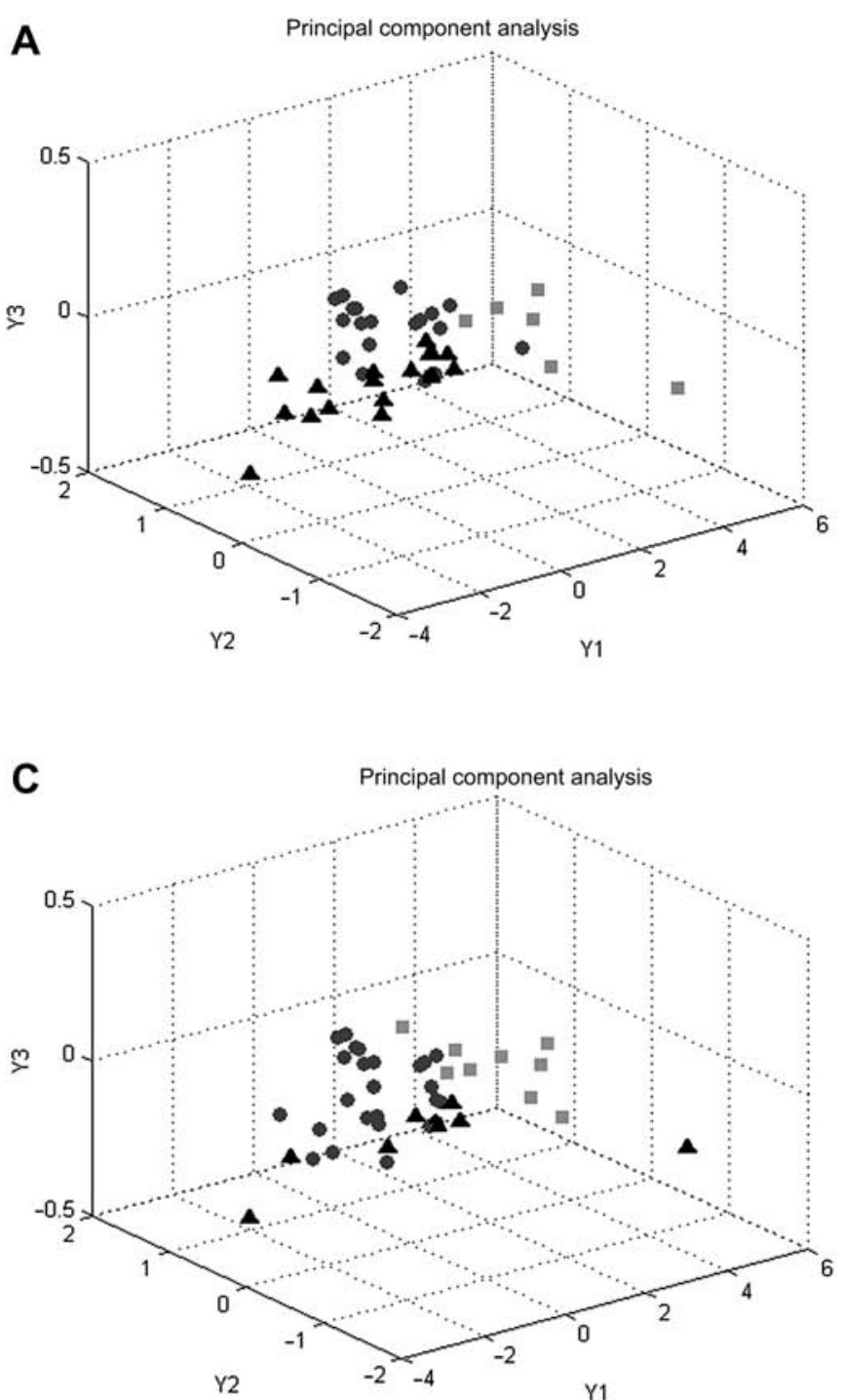

\section{Meaning of the study}

Our findings suggest that lung disease in PCD begins early and that it is better identified at least in school-aged children and adults by the finding of air-trapping or HRCT, rather than
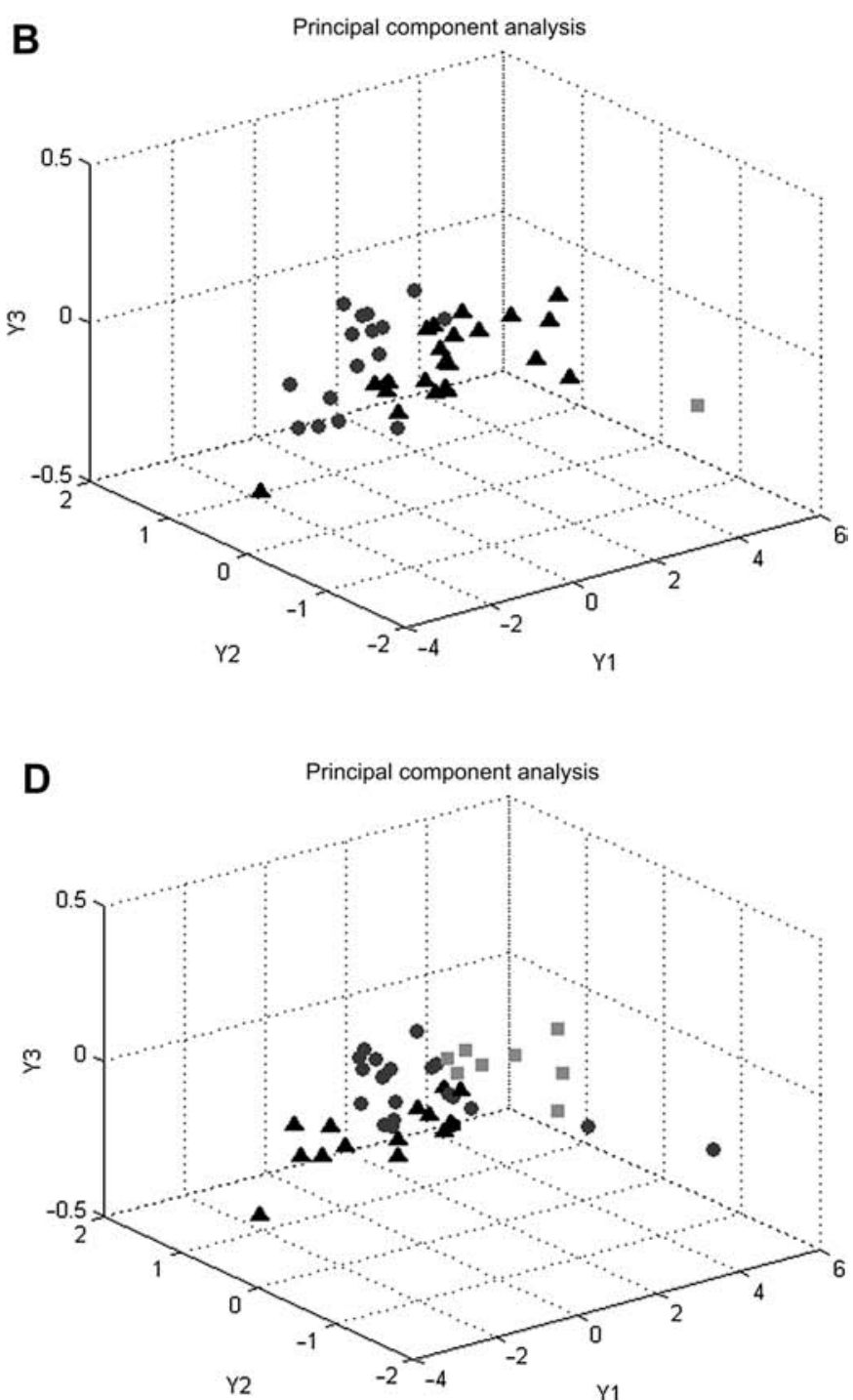

Figure 3 (A) Three-dimensional scatter plots for air-trapping score 1 (triangles), 2 (circles), 3 (squares) in relation to functional residual capacity by plethysmography (FRCpleth), residual volume (RV), total lung capacity (TLC) and RV/TLC ratio. (B) Three-dimensional scatter plots for atelectasis score 1 (triangles), 2 (circles), 3 (squares) in relation to FRCpleth, RV, TLC and RV/TLC ratio. (C) Three-dimensional scatter plots for bronchiectasis extension score 1 (triangles), 2 (circles), 3 (squares) in relation to FRCpleth, RV, TLC and RV/TLC ratio. (D) Three-dimensional scatter plots for total lung impairment score 1 (triangles), 2 (circles), 3 (squares) in relation to FRCpleth, RV, TLC and RV/TLC ratio. 
spirometry. In previous studies of adults and children with PCD, total lung abnormalities and bronchiectasis severity on HRCT were shown to be related to $\mathrm{FEV}_{1}$ and FVC. ${ }^{3} 4$ Our results do not confirm this in a larger series of PCD, even when analysed using a soft computing-based modelling approach, but instead suggest that plethysmography is a much more sensitive test.

CT scanning of the chest has become a popular tool to monitor patients with cystic fibrosis, ${ }^{32}$ a disease that has similarities with PCD. However, no study has evaluated the impact of such monitoring on health outcomes or on clinical decisionmaking in patients with PCD or cystic fibrosis. Moreover, there are concerns on the uncritical use of high-tech medical imaging and on the potential harm of repeated radiological exposures, particularly in children with lifelong diseases. ${ }^{67}$ Consequently, careful thought is needed before requesting repeated chest CT scans. By contrast a detailed evaluation of lung volumes is only limited by the availability of the equipment, and by the ability of the technician to obtain patients' collaboration, but this is usually not a problem in the specialist centres that should care for these patients.

\section{Unanswered questions and future research}

Even though we have not studied very young subjects, from our results it is tempting to speculate that accumulation of secretions, in the early stages of disease, may induce primarily an obstruction in terminal bronchioles which may go undetected for a long period during alveolar growth of the lung. The consequent focal air-trapping during this phase of alveolar growth might lead to impaired septation and decreased elastic recoil as has been observed in animal models. ${ }^{33}$ Early structural changes in the distal airways could explain why we found a lack of correlation between per cent predicted RV and RV/TLC with age, unlike for spirometry, which may reflect slower progressive, age-related damage to large airways. To confirm this hypothesis, data are required from a longitudinal study in patients with PCD from the newborn period, using age-appropriate physiological techniques.

Although our findings show the superior sensitivity of plethysmography over spirometry in detecting HRCT changes in PCD, the findings need to be validated in a second cohort of patients. It would be valuable to compare plethysmography with sophisticated assessments of distal airway function, such as lung clearance index ${ }^{34}$ and distal airway production of exhaled nitric oxide in PCD. ${ }^{35-37}$

Longitudinal data are needed to determine which technique is more sensitive to a change in the patient's condition. Finally, comparisons of plethysmography and spirometry in other airway diseases and in longitudinal studies would be of interest.

In conclusion, in this group who have a high pretest probability of lung damage, ${ }^{27}$ and given the different correlations between the various lung function parameters and HRCTderived lung disease scores we suggest that measurement of lung volumes rather than spirometry may be preferable in the routine clinical management of PCD. Furthermore, this may reduce the number of scans used to monitor disease progression, although this needs testing prospectively. As with all investigations, HRCT should be requested only when the results are going to change the therapeutic strategy in the individual patient, or as part of an ethically approved, focused-research protocol. ${ }^{8}$ Our data represent training results and further work would be required to establish predictive validity, in particular in a second cohort studied longitudinally.

Acknowledgements We wish to thank the Editor and the reviewers for their help in the evaluation of our data.
Contributors M Pifferi: contributed to study design, data evaluation and drafting and revising the submitted manuscript. He had full access to all of the data in the study and he takes full responsibility for the integrity of all of the data and the accuracy of the data analysis. A Bush: contributed to data evaluation and drafting and revising the submitted manuscript. G Pioggia: contributed to data analysis and drafting the submitted manuscript. D Caramella: contributed to data analysis and drafting the submitted manuscript. G Tartarisco: contributed to data analysis and drafting the submitted manuscript. M Di Cicco: contributed to collecting data. M Zangani: contributed to collecting data. I Chinellato: contributed to collecting data. F Maggi performed the statistical analysis. G Tezza: contributed to the reevaluation of all data after reviewers' comments. P Macchia: contributed to study design. A L Boner: contributed to study design, data evaluation and drafting and revising the submitted manuscript.

Funding This research was supported by the Fondazione Carlo Laviosa, Italy.

Competing interests None declared.

Patient consent Obtained.

Ethics approval Ethics approval was provided by Hospital Ethical Committee of Pisa

Provenance and peer review Not commissioned; externally peer reviewed.

\section{REFERENCES}

1. Bush A, Chodhari R, Collins N, et al. Primary ciliary dyskinesia: current state of the art. Arch Dis Child 2007;:92:1136-40.

2. Rossi UG, Owens CM. The radiology of chronic lung disease in children. Arch Dis Child 2005:90:601-7.

3. Kennedy MP, Noone PG, Leigh MW, et al. High-resolution CT of patients with primary ciliary dyskinesia. AJR Am J Roentgenol 2007;188:1232-8.

4. Santamaria F, Montella S, Tiddens HA, et al. Structural and functional lung disease in primary ciliary dyskinesia. Chest 2008;134:351-7.

5. Barbato A, Frischer T, Kuehni CE, et al. Primary ciliary dyskinesia: a consensus statement on diagnostic and treatment approaches in children. Eur Respir $J$ 2009;34:1264-76.

6. Brody AS, Frush DP, Huda W, et al. Radiation risk to children from computed tomography. Pediatrics 2007;120:677-82.

7. Brenner DJ, Hall EJ. Computed tomography-an increasing source of radiation exposure. N Engl J Med 2007;357:2277-84.

8. Fauroux B, Tamalet A, Clément A. Management of primary ciliary dyskinesia: the lower airways. Paediatr Respir Rev 2009:10:55-7.

9. Stănescu D. Small airways obstruction syndrome. Chest 1999;116:231-3.

10. Tiddens HA, Donaldson SH, Rosenfeld M, et al. Cystic fibrosis lung disease starts in the small airways: can we treat it more effectively? Pediatr Pulmonol 2010;45:107-17

11. Green K, Buchvald FF, Marthin JK, et al. Ventilation inhomogeneity in children with primary ciliary dyskinesia. Thorax 2012;67:49-53.

12. Marthin JK, Petersen N, Skovgaard LT, et al. Lung function in patients with primary ciliary dyskinesia: a cross-sectional and 3-decade longitudinal study. Am J Respir Crit Care Med 2010;181:1262-8.

13. Rutland J, Cole PJ. Non-invasive sampling of nasal cilia for measurement of beat frequency and study of ultrastructure. Lancet 1980:2:564-5.

14. Pifferi M, Cangiotti AM, Ragazzo V, et al. Primary ciliary dyskinesia: diagnosis in children with inconclusive ultrastructural evaluation. Pediatr Allergy Immunol 2001:12:274-82

15. Carlén B, Stenram U. Ultrastructural diagnosis in the immotile cilia syndrome. Ultrastruct Pathol 1987:11:653-8.

16. Pifferi M, Montemurro F, Cangiotti AM, et al. Simplified cell culture method for the diagnosis of atypical primary ciliary dyskinesia. Thorax 2009:64:1077-81.

17. American Thoracic Society. Standardization of spirometry: 1994 update. Am J Respir Crit Care Med 1995;152:1107-36.

18. American Association for Respiratory Care. Clinical Practice Guideline: static lung volumes: 2001 revision \& update. Respir Care 2001;46:531-9.

19. Zapletal A, Samánek M, Paul T. Lung Function in Children and Adolescents. Methods, Reference Values. Basel, Switzerland: S Karger AG, 1987

20. Coates AL, Peslin R, Rodenstein D, et al. Measurement of lung volumes by plethysmography. Eur Respir J 1997;10:1415-27.

21. Bhalla $\mathbf{M}$, Turcios $\mathrm{N}$, Aponte $\mathbf{V}$, et al. Cystic fibrosis: scoring system with thinsection CT. Radiology 1991;179:783-8.

22. Pifferi M, Caramella D, Bulleri A, et al. Pediatric bronchiectasis: correlation of HRCT ventilation and perfusion scintigraphy, and pulmonary function testing. Pediatr Pulmonol 2004;38:298-303.

23. Jamshidi M. Tools for intelligent control: fuzzy controllers, neural networks and genetic algorithms. Philos Transact A Math Phys Eng Sci 2003;361:1781-808.

24. Shaw PJ. Multivariate Statistics for the Environmental Sciences. London, UK: Hodde Arnold, 2003.

25. Dibley MJ, Goldsby JB, Staehling NW, et al. Development of normalized curves for the international growth reference: historical and technical considerations. Am J Clin Nutr 1987; 46:736-48. 
26. Pifferi M, Caramella D, Ragazzo V, et al. Low-density areas on high-resolution computed tomograms in chronic pediatric asthma. J Pediatr 2002;141:104-8.

27. Jain K, Padley SP, Goldstraw EJ, et al. Primary ciliary dyskinesia in the paediatric population: range and severity of radiological findings in a cohort of patients receiving tertiary care. Clin Radiol 2007;62:986-93.

28. Davis SD, Brody AS, Emond MJ, et al. Endpoints for clinical trials in young children with cystic fibrosis. Proc Am Thorac Soc 2007;4:418-30.

29. Noone PG, Leigh MW, Sannuti A, et al. Primary ciliary dyskinesia: diagnostic and phenotypic features. Am J Respir Crit Care Med 2004;169:459-67.

30. Dakin CJ, Numa $\mathrm{AH}$, Wang $\mathrm{H}$, et al. Inflammation, infection, and pulmonary function in infants and young children with cystic fibrosis. Am J Respir Crit Care Med 2002;165:904-10.

31. Brown DE, Pittman JE, Leigh MW, et al. Early lung disease in young children with primary ciliary dyskinesia. Pediatr Pulmonol 2008;43:514-16.
32. Robinson TE. Computed tomography scanning techniques for the evaluation of cystic fibrosis lung disease. Proc Am Thorac Soc 2007:4:310-15.

33. Srinivasan G, Bruce EN, Houtz PK, et al. Dexamethasone-induced changes in lung function are not prevented by concomitant treatment with retinoic acid. Am J Physio Lung Cell Mol Physiol 2002;283:L275-87.

34. Gustafsson PM, De Jong PA, Tiddens HA, et al. Multiple-breath inert gas washout and spirometry versus structural lung disease in cystic fibrosis. Thorax 2008;63:129-34.

35. Corbelli R, Bringolf-Isler B, Amacher A, et al. Nasal nitric oxide measurements to screen children for primary ciliary dyskinesia. Chest 2004;126:1054-9.

36. Mateos-Corral D, Coombs R, Grasemann $\mathrm{H}$, et al. Diagnostic value of nasal nitric oxide measured with non-velum closure techniques for children with primary ciliary dyskinesia. J Pediatr 2011;159:420-4.

37. Pifferi M, Bush A, Maggi $F$, et al. Nasal nitric oxide and nitric oxide synthase expression in primary ciliary dyskinesia. Eur Respir J 2011;37:572-7.

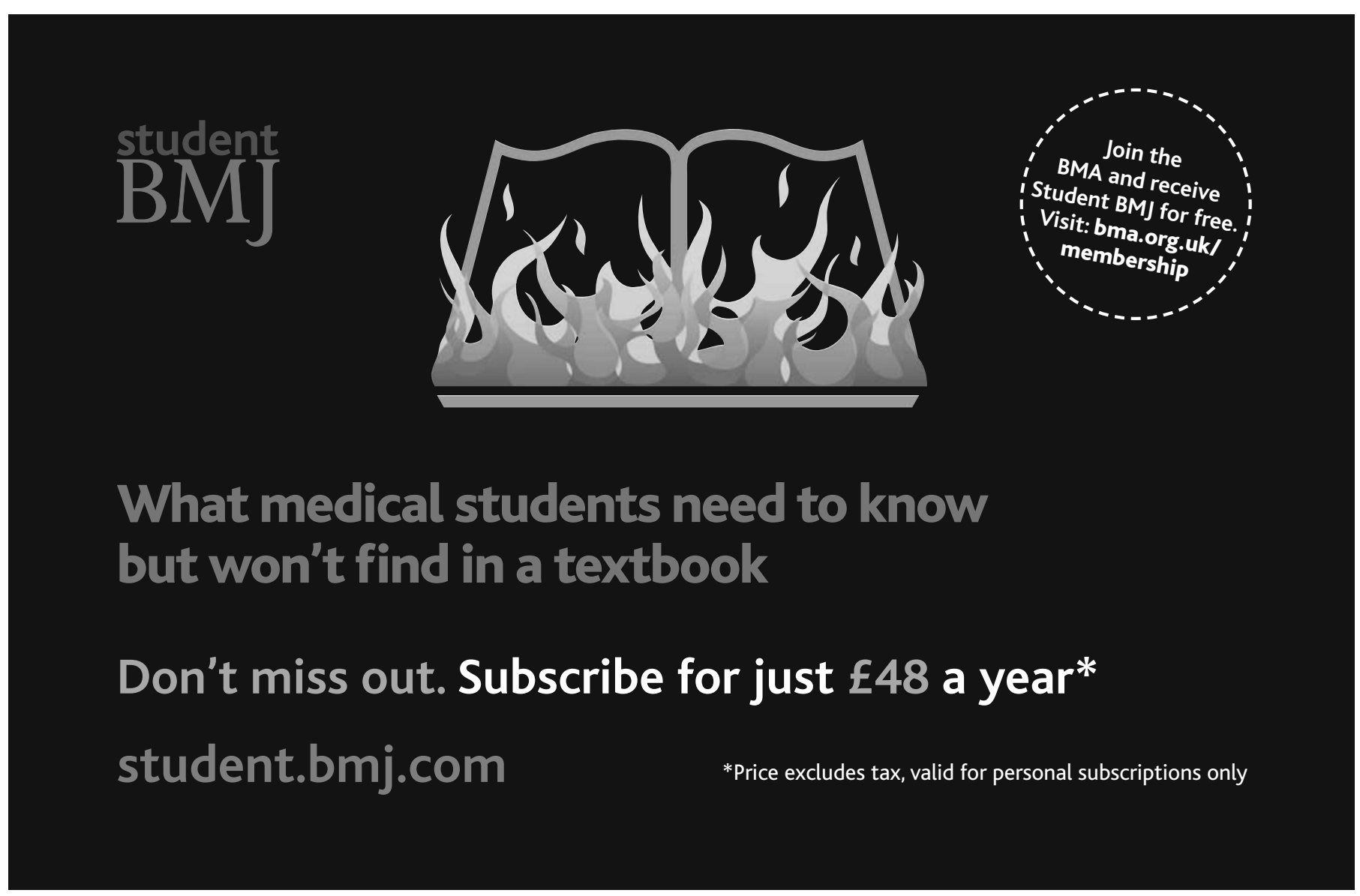

